\title{
Sonic Gestures Applied to a Percussive Dialogue in TanGram Using Wii Remotes
}

\author{
Carlos D. Perales, Cristina Portalés, and Francisco Sanmartín \\ Universidad Politécnica de Valencia, Camino de Vera, s/n. 46022 Valencia. Spain, \\ carpece@doctor.upv.es, criporri@upvnet.upv.es, \\ frasanpi@pin.upv.es
}

\begin{abstract}
TanGram, is an original music score composed by Carlos D. Perales for Percussion Quartet and Nintendo Wii Remotes used as a wireless interface for Live Electronics. This paper examines how this composition explores exiting research in interaction and communication between instruments and digital sound processing discourse to produce a unique music mixed-media score, to explore the sonic possibilities of a multi-timbrical instrument with the integration of the visual gestures of an accessible device using processes of real-time gestural mimesis.
\end{abstract}

Keywords: Music, Electroacoustic, Live Electronics, Percussion.

\section{Real Time Discourse Based on Digital Sound Processes}

\subsection{Designing Behaviour}

The concept of real time processes has been extensively explored and discussed in composition and improvisation environments [1]. However the integration of wearable devices on percussion performers as an extension of them, especially in percussion pieces, opened a scope for another twist, as methodologies and research from the field of Acousmatic Music and performance explorations were introduced. To explain the integration between the wireless interface and the traditional use of mallets in percussion instruments, we start with the music score, which is designed to precise both uses.

Each percussionist has attached to his arm the Wii remote control in order to achieve a new parameter in movements and gestures (Fig. 1). Because the application runs as an organic sequence of events (DSP processes) and determines a collaborative distribution for the performers, a standalone Max MSP patch [2] was designed as a start point in the managing of processes. In order to connect the Wii dates with Max MSP a specific library object, named aka.wiiremote [3] have been used. This object allows a stable data transfer via Bluetooth. Each percussionist activate/deactivate their own events list using the ' $\mathrm{A}$ ' button on Wii remote control.

\subsection{From Gestures to Notated Sound}

Although some electroacoustic composers have developed some conclusions of gestural analysis of sound for the analysis of live electronics performances, my intention was to reorient this findings towards writing a musical score that bring the freedom to 

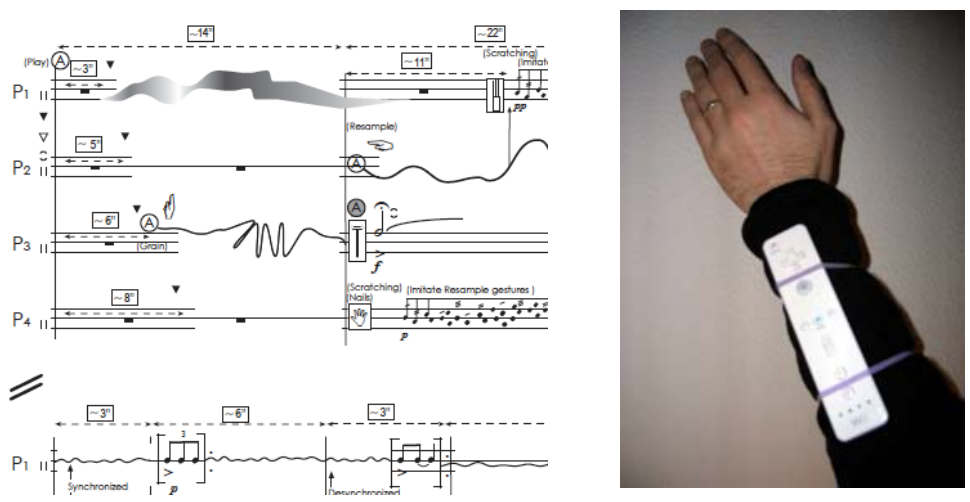

Fig. 1. Score excerpt - Wii remote

the performer to control and vary the responses to the other performers. I wanted to describe in an precise way, how after solving the tilt behaviour puzzle of acceleration in the $\mathrm{x}$ or $\mathrm{y}$ axes, 'Kontakte Percussion Group' [4], the performers who commissioned this electro-acoustic score, had therefore to make extensive use of their collaboration and the different performances experiences by calling on their sonic memory and creativity. After activate any process, they had to listen and interiorize their own movements for the necessary coordination between rhythms, mallets, gestures and feedback dialogue.

During this process of integration, which involved microphone experimentation and digital techniques, a sound engineer, who also produced the $\mathrm{CD}$ recording of the piece in winter 2009, assisted the performers.

\section{Tam-Tam Morphology; A Further Step}

\subsection{Score Re-contextualization}

This score is a re-contextualization of existing research in typology and morphology of percussion exploration and concrete sonic objects started by Pierre Schaeffer [5], continued among others by Dennis Smalley [6], and reunited by Lasse Thoresen [7]. Karlheinz Stockhausen made a specific multi-timbrical exploration of the tam-tam in his piece Microphonie.

Anyway I didn't want to build a parallel world of concrete objects on the instruments but a traditional use of tam-tam with all kind of percussion beaters and two concrete sources as adhesive tape and stones curtain (Fig. 2).

The tam-tam is a very common resonator. Obviously I didn't want to make a whole piece based on this parameter, because the electroacoustic give us the chance to explore it as an extension of it sound.

\subsection{Performer Independence}

Normally in electroacoustic pieces with live electronics a technician is required to control the live events and common aspects as gain, balance, etc. As the performers wanted to be 


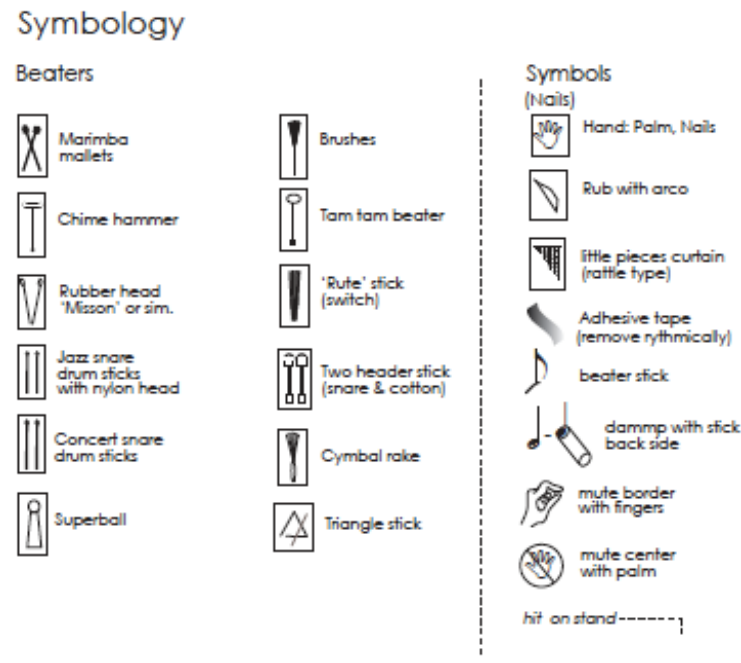

Fig. 2. Beater symbology

independents on playing the score, the patch designed is almost entirely controlled from the Wii buttons. The up and down buttons control the stereo gain. The 'A' button triggers each process saved from a coll. A matrix orient the incoming dates from each Wii to a specific DSP process (Fig. 3). Also the four microphones are activates or deactivated depending on other similar matrix reading from a coll list of events.

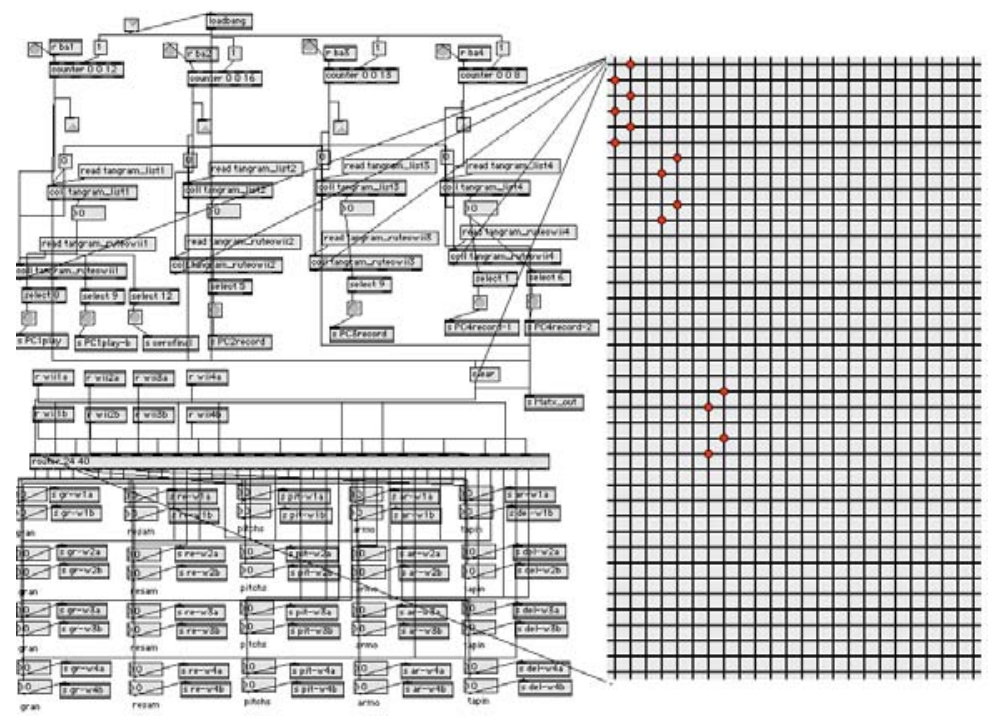

Fig. 3. Max MSP Matrix 


\subsection{Digital Behaviour}

The musical score, including the computer part (real time processes) is a path iteration of probabilities based on Chinese TanGram game and its geometric pieces. Concepts of sound spectrum, spectral brightness, pulse, multi directionality and harmonicity act as parallel layer with the tam-tam live sound.

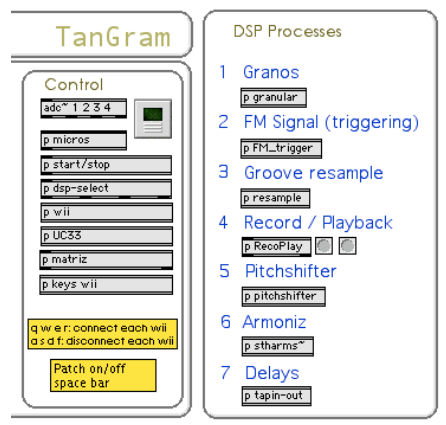

Fig. 4. DSP Processes

Seven pieces configures this easy Chinese game. Therefore, seven digital processes, seven sections and seven rhythmic motives where established for the score (Fig. 4), aiming to find beauty and musical expression in the matches and divergences between the original sound of the tam-tams and the mimetic response of the electronic discourse.

\subsection{Notated Methodology}

Performers need clear instructions to play around the Tam surface. A 'trigram' was established meaning the three points to hit or rub on it surface. Additional symbols were attached on score to illustrate the stand support. In order to control the damping of the tam-tam resonation, I define whether performers have to stop the resonation sharply, flatly or leave it sounding (Fig. 5). The way that they damp is a free option. In Fig. 6, the tilt of performer 1 is synchronized between the other performers.

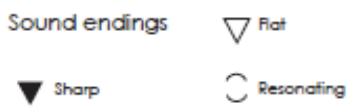

Fig. 5. Damping marks

Also damping with the left hand in two ways modifies each beater attack: by holding the edge or covering the centre area with the hand's palm. Dynamics are fortissimo (fff) to pianissimo (ppp) and some dramatic pauses are used. 


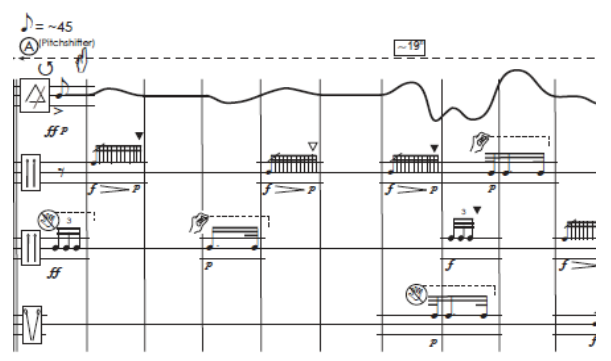

Fig. 6. Score excerpt

\subsection{Rhythmic Nature}

As a percussion piece the articulation and structure is entirely driven by rhythm. Only some uses of bowing and rubber techniques act as an exception of the whole piece. However, it is not just understood as rhythm in musical terms but as pace, informed by Tarkowskij's methodology [8], which is not focused on the temporal editing, but on the rhythm of the scenes (sculpting in time); in TanGram terms, it refers to the pace of different sonic scenes with a characteristic typology. By the way each electroacoustic process requires a specific tempo, so the rhythm is adapted to each response.

\section{Conclusions}

This paper has discussed the implementation aspects of gestural behaviour in a mixed-media piece for percussion and lives electronics called TanGram. With the introduction of the autonomy given by the Wii remote control via Max MSP patch, in this context, the author proposes a new route for creative expression, informed by Tarkowskij's ideas and methodologies in film and scene montage [9], when exposing the Ideal to its Mirror. Similarly, the score utilizes vocabulary and specific typology to precisely notate gestures and movement, generated and transformed employing computer and recording techniques. An explanation of the existing methodology to read and interpret the score leads to some detailed examples about how to notate in time concepts such as movement gestures, length variations and others, which belong to the electroacoustic vocabulary.

Acknowledgment. 'Kontakte Grup the Percussió' commissioned TanGram. The score and research behind it was realized between 2008 and 2009.

\section{References}

1. Percussion in the New-Old World. Wilfrid Mellers. The Musical Times, 133(1795), 445447 (September 1992)

2. Puckette, M., Zicarelli, D.: Max MSP software, http: / /www . cycling74 . com 
3. Aka Universal Objects for Max MSP 4.6, http: / /www. iamas.ac.jp/ aka/max/

4. Kontakte Grup de Percussió, http://www.kontakte-percusion.com (last visited January 20, 2009)

5. Schaeffer, P.: Typology and Morphology of Sonic Objects. Traite des objets musicaux. Essay Interdisciplines. Nouvelle edition, Seuil (1966)

6. Smalley, D.: Spectromorphology: Explaining sound-shapes. Organised Sound 2(2), 107126 (1997)

7. Thoresen, L.: Spectromorphological analysis of sound objects: an adaptation of Pierre Schaeffer's typomorphology. Organised Sound archive 12(2), 129-141 (2007)

8. Dempsey, M.: Andrej Tarkowskij's films. Lost Harmony: Tarkovsky's "The Mirror" and "The Stalker". Film Quarterly 35(1), 12-17 (Autumn 1981)

9. Deltcheva, R., Vlasov, E.: Back to the House II: On the Chronotopic and Ideological Reinterpretation of Lem's Solaris in Tarkovsky's Film. Russian Review 56(4), 532-549 (1997); Published by: Blackwell Publishing on behalf of The Editors and Board of Trustees of the Russian Review 